\title{
A randomised double-blind placebo-controlled trial investigating the behavioural effects of vitamin, mineral and $n-3$ fatty acid supplementation in typically developing adolescent schoolchildren
}

\author{
Jonathan D. Tammam ${ }^{1 *}$, David Steinsaltz ${ }^{2}$, D. W. Bester ${ }^{2}$, Turid Semb-Andenaes ${ }^{1}$ and John F. Stein ${ }^{1}$ \\ ${ }^{1}$ Department of Physiology, Anatomy and Genetics, University of Oxford, Oxford, OX1 3PT, UK \\ ${ }^{2}$ Department of Statistics, University of Oxford, Oxford, OX1 3TG, UK
}

(Submitted 19 March 2015 - Final revision received 18 September 2015 - Accepted 6 October 2015 - First published online 17 November 2015)

\section{Abstract}

Nutrient deficiencies have been implicated in anti-social behaviour in schoolchildren; hence, correcting them may improve sociability. We therefore tested the effects of vitamin, mineral and $n-3$ supplementation on behaviour in a 12-week double-blind randomised placebocontrolled trial in typically developing UK adolescents aged 13-16 years ( $n$ 196). Changes in erythrocyte $n-3$ and 6 fatty acids and some mineral and vitamin levels were measured and compared with behavioural changes, using Conners' teacher ratings and school disciplinary records. At baseline, the children's PUFA ( $n-3$ and $n-6)$, vitamin and mineral levels were low, but they improved significantly in the group treated with $n$-3, vitamins and minerals $(P=0 \cdot 0005)$. On the Conners disruptive behaviour scale, the group given the active supplements improved, whereas the placebo group worsened $(F=5.555, d=0.35 ; P=0.02)$. The general level of disciplinary infringements was low, thus making it difficult to obtain improvements. However, throughout the school term school disciplinary infringements increased significantly (by $25 \%$; Bayes factor $=115$ ) in both the treated and untreated groups. However, when the subjects were split into high and low baseline infringements, the low subset increased their offences, whereas the high-misbehaviour subset appeared to improve after treatment. But it was not possible to determine whether this was merely a statistical artifact. Thus, when assessed using the validated and standardised Conners teacher tests (but less clearly when using school discipline records in a school where misbehaviour was infrequent), supplementary nutrition might have a protective effect against worsening behaviour.

Keywords: n-3 Supplementation: Micronutrient supplementation: Behaviour: Adolescents: PUFA

The relationship between diet and behaviour is complex and poorly understood. Childhood malnutrition has been associated with behavioural problems such as in attention, aggression and impaired socialisation $^{(1)}$. Individuals with micronutrient and $n-3$ PUFA deficiencies may be particularly prone to such anti-social behaviour $^{(2)}$ and aggression ${ }^{(3,4)}$.

EPA and DHA are particularly important for brain health. EPA is converted into many important signalling eicosanoids, and DHA is a major constituent of neuronal and glial cell membranes. Thus, the early development of the brain is vulnerable to deficiencies of these fatty acids in the diet ${ }^{(4,5)}$. In addition, a high $n-6: n-3$ ratio may lead to an overproduction of $n-6$ eicosanoids ${ }^{(6)}$, which can adversely affect neurotransmission ${ }^{(7)}$. Many studies use a single-nutrient intervention, an approach that has methodological and generalisability limitations. A recent review suggests that a multi-nutrient approach can ameliorate anti-social behaviour as well as a range of psychiatric symptoms ${ }^{(8)}$. From a cognitive health point of view, the metabolic processes governing neurotransmitter production and utilisation require cofactors for enzyme action, which generally comprise vitamins and minerals ${ }^{(9)}$. It is reasonable to suggest that an appropriate nutrient intervention for improvement of cognitive health and behaviour would be one that contains a broad range of vitamins, minerals and essential fatty acids (EFA) ${ }^{(10,11)}$. Parletta et al. ${ }^{(12)}$ provide mechanistic insights for the role of nutrients in cognitive health. Increasing $n-3$ PUFA and micronutrient intake may therefore be an effective way to improve brain function and behaviour.

Supplementary multinutrient intervention has been shown to significantly reduce disciplinary transgressions by schoolchildren in a working class environment ${ }^{(13)}$ and by young prisoners ${ }^{(14-16)}$. However, studies in typically developing adolescent schoolchildren are lacking. In 8- to 10-year-old schoolchildren, little influence of supplementation was seen on cognitive outcomes ${ }^{(17)}$, but a small behavioural effect was detected, which the authors concluded was equivocal. However, $n-3$ supplementation (in fruit juice) in 8- to 16-year-old community-dwelling Mauritanian children led to reductions in parent-rated behaviour problems ${ }^{(18)}$. Whether this result would apply to UK children is not known.

Abbreviations: ADHD, attention-deficit hyperactivity disorder; EFA, essential fatty acid.

* Corresponding author: J. D. Tammam, fax +44 1865 272478, email jonathan.tammam@dpag.ox.ac.uk 
Many trials focus on children with neurodevelopmental disorders - for example attention-deficit hyperactivity disorder (ADHD). Older studies have implicated poor diets in anti-social behaviour ${ }^{(19-21)}$ and more recently significant behavioural improvements have been reported after supplementation ${ }^{(22-26)}$. However, some studies ${ }^{(27,28)}$ found no improvement in children with ADHD after supplementing with DHA alone, whereas supplementation with broad-spectrum vitamins and minerals in adults with ADHD did improve symptoms ${ }^{(29)}$. But EFA supplementation produced no changes in aggression, hostility and irritability in trials using normal volunteers ${ }^{(30,31)}$. In studies that utilised micronutrients (in the absence of EFA), Fe supplementation led to improvements, $v$. placebo, in ADHD measures in young children aged between 5 and 8 years ${ }^{(32)}$ and Mg supplementation with and without vitamin $\mathrm{B}_{6}$ in children with ADHD, respectively, led to improvements in attention and aggression $^{(33)}$ and reductions in hyperactivity ${ }^{(34)}$. Furthermore, multivitamin supplementation in young adults led to improvements in serum vitamin levels and measures of behaviour such as agreeability and composure ${ }^{(35)}$. Thus, there may be important potential for a multinutrient approach in treatment of neurobehavioural conditions ${ }^{(8)}$. However, multivitamin supplementation in addition to PUFA provided no additional benefit over PUFA alone ${ }^{(25)}$, but the authors suggest that the multivitamin dose might have been insufficient to provide treatment efficacy.

As regards adolescent schoolchildren, the evidence is highly heterogeneous in terms of study design, population, outcome measure, nutrient intervention and scientific rigour. Nevertheless, the evidence for improved behaviour after nutritional supplementation in correctional facilities is consistent. Hence, the balance of evidence would suggest a positive role for multiple vitamins, minerals and EFA in both neuro-impaired and neurotypical individuals. Therefore, a similar intervention may improve behavioural outcomes in other contexts, such as in schools.

We therefore decided to study adolescents in an underprivileged area in England. The UK Low Income Diet and Nutrition Survey ${ }^{(36)}$ showed that individuals in lower socioeconomic groups are likely to have significant micronutrient deficiencies. The UK National Diet and Nutrition Survey suggests that in particular $n-3$ PUFA intake from oily fish may be well below recommendations ${ }^{(37)}$. There is also evidence to suggest that cognitive improvements can be gained from supplements even in individuals enjoying adequate nutritional status ${ }^{(35,38)}$.

Here we describe a double-blind randomised placebocontrolled nutritional intervention trial to compare the behavioural effects of vitamin, mineral, DHA and EPA supplements with placebo in typically developing 13- to 16-year-old adolescent schoolchildren in the UK. We used multi-nutrient supplementation as a proxy for an improved diet, which, although it cannot offer mechanistic insights, can assess the efficacy of treatment. Our intention was not to ascertain the relative importance of individual nutrients, rather to provide treatment with multiple nutrients at physiological doses in a population that is likely to be experiencing broad-spectrum low micronutrient intake.

\section{Methods}

This was a parallel-group, randomised, double-blind, placebocontrolled study. The aim was to assess the impact of micronutrient and EFA supplementation on behaviour in adolescent schoolchildren aged between 14 and 15 years.

\section{Participants}

A large comprehensive secondary school (Robert Clack School), which educates 11- to 18-year-old boys and girls, hosted this study. The school, which provided great support, is located in the East End of London borough of Barking and Dagenham. A large proportion of adults in the surrounding area are economically inactive and hold no formal educational qualifications. Consequently, many children face adverse environments and poor nutrition, which can manifest as challenging behaviour at school.

Typically developing schoolchildren from years 9 and 10 (predominantly aged 14-15 years, with a minority aged 13 and 16 years) were invited to participate in the trial. Its purpose and requirements were fully explained before recruitment took place. Pupils ( $n$ 196) were recruited over four school terms and randomised to either active treatment or placebo for a single term (approximately 3 months). Exclusion criteria were major medical disorders (including ADHD/ADD), prescribed psychoactive medication, current consumption of multivitamin and/or fish oil supplements, allergy to fish and eating fish $>2$ times/week. Participants were recruited and allocated according to the flowchart in Fig. 1.

\section{Ethics}

The study was conducted according to the guidelines of the Declaration of Helsinki, and all procedures involving human subjects were approved by the South West Research Ethics Committee (Ref: 10/H0206/13). Written informed consent was obtained from all subjects' parents. Children provided verbal assent, before initial testing, which was witnessed and formally recorded. Participants were able to leave the study at any time without reason. The trial was registered at Current Controlled Trials in the same month, immediately before recruitment (http://www.controlled-trials.com) ISRCTN03817376.

\section{Power and sample size}

We computed power by performing the same statistical analysis that we would apply to the real data, on 1000 simulated data sets. If the average rate of offences is $0.05 /$ subject per $\mathrm{d}$ (corresponding to 3.85 offences over the 77 mean d of the study), the power to detect an improvement of $28 \%$ (corresponding to $\alpha_{A}=0.25$ in our statistical model) at a threshold corresponding to 0.05 significance would be about $0 \cdot 80$. (We took the variance parameter - measuring the variation among children in their offence rates - to be $0 \cdot 0040$, but substantial changes in this parameter were found to produce only minor changes in the power.) If the mean offence rate were only 0.02 , the power would drop to $0 \cdot 454$; if the mean offence rate were raised to $0 \cdot 10$, the power would go up to 0.97 . 


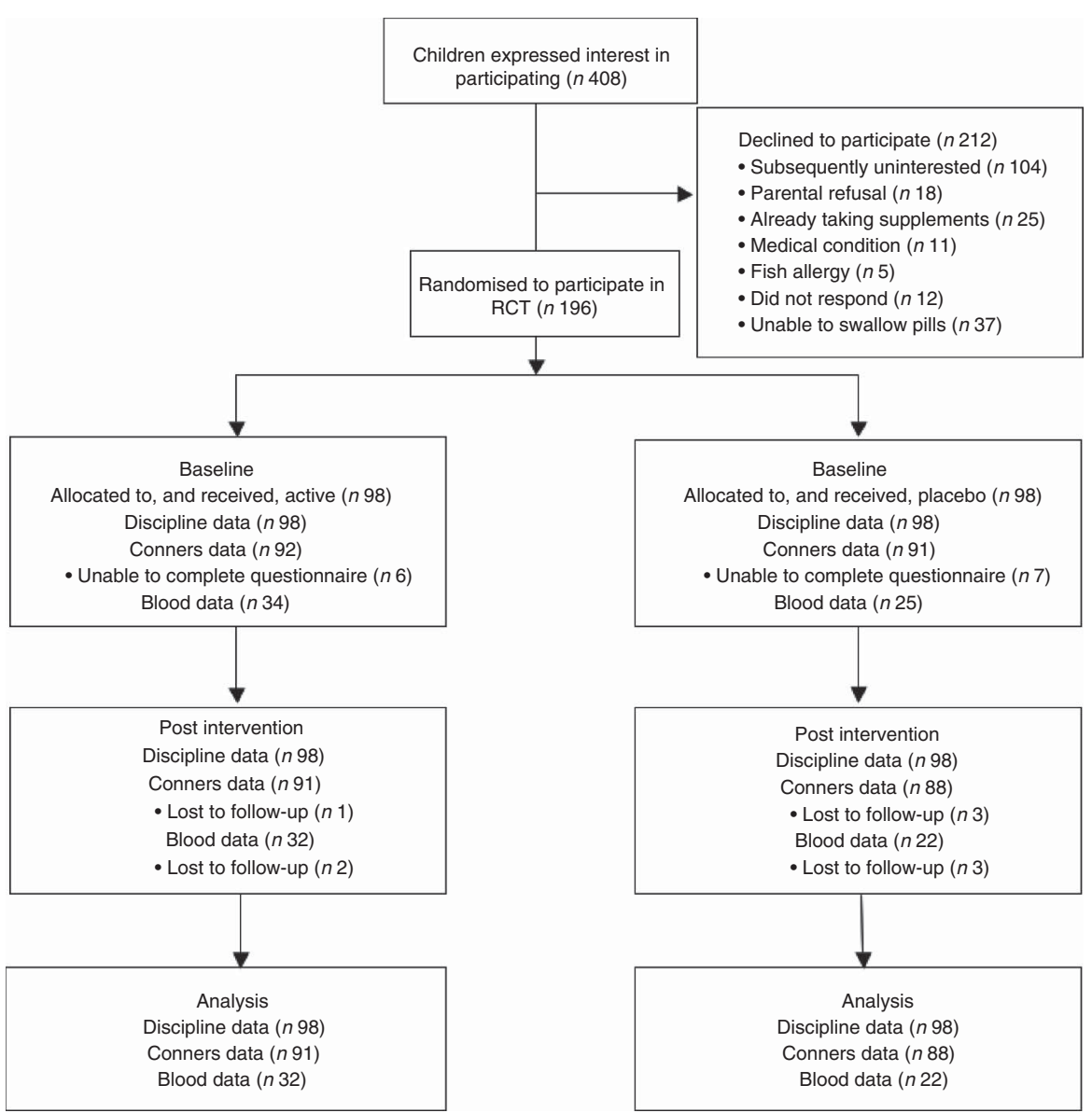

Fig. 1. Flow of participants through the study. RCT, randomised controlled trial.

\section{Intervention}

Active daily treatment was one tablet containing multivitamins/ minerals and two capsules containing $n$-3 PUFA (WellTeen Plus; Vitabiotics Ltd). The potency of the active vitamin and mineral supplementation is described in online Supplementary Table S1 and compared with UK reference nutrient intake ${ }^{(39)}$ where applicable. The active PUFA supplement contained EPA $(165 \mathrm{mg})$ and DHA $(116 \mathrm{mg})$, and thus the children consumed $281 \mathrm{mg} / \mathrm{d}$ combined EPA plus DHA. The placebo tablets contained dicalcium phosphate with potato starch and the placebo capsules contained sunflower oil, which were colour-, odour- and flavourmatched with the active tablets and capsules. Active and placebo allocation was carried out according to a randomisation schedule provided by the manufacturer of the supplements and allocated to participants on a per-cohort basis. Supplements were dispensed from individually labelled compartment trays, which had minimal risk of contamination. Trays remained locked at all times, other than during the lunchtime supplementation period. Researchers, participants and school staff were blind to treatment allocation. Before commencing supplementation and at the end of the study, participants were asked whether they thought they had received active or placebo treatment. In addition, teachers who completed the behaviour questionnaire were asked whether they thought the participant had received active or placebo treatment.
During the 12-week intervention period, participants were given supplements with a cup of water during the lunch break during school days. Compliance was monitored by observing swallowing, and it was recorded on a register. This greatly reduced the risk of participants discarding or swapping pills. At weekends and other planned absences, participants were provided with an appropriate amount of supplements with instructions to consume them on a daily basis. They confirmed compliance on a tick-box form.

\section{Outcome measures}

Baseline dietary intake. Participants were asked to keep a 7-d food diary by detailing everything consumed in the week before commencing the study. Completed diaries were analysed for nutrient composition using Dietplan 6.5 (Forestfield), and baseline differences between treatment groups were calculated to ascertain the level of matching of diet in participants between groups.

Erythrocyte essential fatty acid and micronutrient analysis. Venous blood samples were taken from the fifty-three participants who consented to provide blood. A phlebotomist collected $10 \mathrm{ml}$ of blood into a labelled EDTA tube (Becton Dickinson). 
Tubes were immediately transferred to a chilled transport box and delivered to Oxford for overnight storage at $4^{\circ} \mathrm{C}$ in a blood fridge. Samples were then spun at $4^{\circ} \mathrm{C}$ at $2000 \mathrm{rpm}$ for $20 \mathrm{~min}$ to separate the various fractions. Plasma and erythrocyte fractions were separated and stored in cryovials before long-term storage at $-80^{\circ} \mathrm{C}$. The PUFA EPA, DHA, total $n-3$, total $n-6, n-6: n-3$ ratio and $n-3+n-6$ ( $n-3$ index) were analysed in erythrocytes according to the method of Bell et al. ${ }^{(40)}$. Additional micronutrients analysed in plasma were Fe, ferritin, folate, vitamin C and vitamin D. These micronutrients were analysed at Medical Research Council Human Nutrition Research (Cambridge) according to the following methods. Fe was measured colourimetrically using the Siemens Dimension Xpand; the assay uses Ferene ${ }^{\circledR}$ as chromophore. Total Fe-binding capacity is measured by saturating all Fe-binding sites with added ferric salt and then releasing it and measuring the released Fe using the same reaction. Ferritin was measured using the Siemens Dimension automated immunoassay, which uses a $\mathrm{CrO}_{2}$ microparticle solid phase and $\beta$-galactosidase label. Folate was measured on the Siemens Advia Centaur, using a competitive immunoassay with a fluorescence end point. Vitamin C (ascorbic acid) was measured in plasma stabilised with metaphosphoric acid. Ascorbic acid in the sample is converted to dehydroascorbic acid by ascorbate oxidase; this is then coupled to o-phenylenediamine to form quinoxaline, which can be quantified by fluorescence. 25-Hydroxyvitamin D (25(OH)D) was measured using the Liaison Analyser (Diasorin), which uses a direct non-extraction chemiluminescent immunoassay method. The antibody is immobilised on magnetisable particles and the competing antigen is a $25(\mathrm{OH}) \mathrm{D}-$ isoluminol conjugate. We had intended to analyse additional nutrients from venous blood samples; however, as a result of technical difficulties in processing the blood samples, this was unfortunately not possible, and this anomaly is reflected in the discussion.

Conners teacher assessment of behaviour. Teachers rated pupil behaviour using the Conners Clinical Index Teacher Rating Scale (a twenty-four-question test from the battery of Conners Comprehensive Behaviour Rating Scales) ${ }^{(41)}$, first immediately before capsule administration and a second time towards the end of the final week of intervention. As measures of child behaviour and social and psychological functioning, Conners scales are age-standardised and have been shown to be valid and reliable. T-scores (mean 50 (SD 10)) were calculated, with higher scores indicating increased severity of behavioural symptoms. These tests have previously been used in $n-3$ fatty acid supplementation trials in schools ${ }^{(42)}$. Although the Conners' test provides five subscales, we expected the disruptive behaviour disorder subscale to show the greatest changes, and thus our analysis focused on this score.

School discipline records. Negative behaviours resulting in disciplinary action were logged in the school's self-designed databases on a daily basis (e.g. at the Pupil Referral Unit or Learning Support Centre and additional databases). Databases provided logs of, inter alia, dates, times and reason for referral for example, disruptive behaviour, aggression, tardiness and so on. School records were reviewed for disciplinary incidents during the treatment period. Historic records were obtained from the previous school term to provide baseline discipline information. Number (and rate) of reports over a period of time was the outcome measure. Similar indicators of behaviour have been used in nutrient supplementation trials in schools and prisons ${ }^{(15,16)}$.

\section{Statistical analysis}

Other than for the discipline data analyses (see below), SPSS version 19 for Mac (SPSS Inc.) was used. In all cases, two-tailed significance values are given and $P \leq 0.05$ was considered statistically significant.

Baseline measurements and allocation of treatment guesses. Age, cohort, year group and treatment allocation guesses are expressed as mean and standard deviations. Comparisons were performed using the $\chi^{2}$ test. Baseline dietary data, expressed as mean and standard deviations, were compared using independent samples $t$ tests.

Erythrocyte PUFA and micronutrient status. Within- and between-group analyses of PUFA and micronutrient concentrations were carried out on a per-protocol basis, excluding participants if follow-up data were missing. Paired $t$ tests were performed on each subscale to evaluate the effect of intervention on the active or placebo groups. Furthermore, a one-way ANCOVA was performed to calculate post-intervention between-group differences while controlling for baseline differences. The independent variable was the group allocation, the dependent variable was post-treatment PUFA concentrations and the covariate was baseline PUFA concentrations.

Conners teacher assessment. Within- and between-group analyses of Conners subscale $T$-scores were carried out on a per-protocol basis, by excluding participants if follow-up data were missing. Paired $t$ tests were performed on each subscale to evaluate the change in mean score within the active or placebo group. A one-way ANCOVA was also performed to estimate the effect of the treatment on this change, while controlling for baseline differences. The independent variable was the group allocation, the dependent variable was post-treatment score and the covariate was baseline score. Differences between groups in calculated change (post-pre) scores were also analysed by two-sample (paired within individuals) $t$ tests. The results for all tests are reported for all five subscales, but the disruptive behaviour subscale was our primary interest.

School discipline records - full analysis. All participants were included in the analysis of school disciplinary records, on an intent-to-treat (ITT) basis. We analysed the treatment effect on disciplinary offences in the schoolchildren within a Bayesian modelling framework. This means that the treatment effect is posed as an unknown random quantity whose distribution is to be inferred from the data. We made the standard simplifying assumption that each individual's offences form a Poisson process, occurring at a constant rate within a period (baseline or treatment), although the rate differs for different individuals, and may change from the baseline to the treatment period. 
A detailed model description, along with estimation techniques, can be found in the online Supplementary Material. The crucial estimate of effect size, the proportional change in the offence rate for those receiving the treatment, is therefore $e^{\alpha A}$. Negative values of $\alpha_{A}$ would correspond to our prediction that active treatment would reduce an individual's offence rate; positive values of $\alpha_{A}$ mean that active treatment increased the offence rate.

To test the significance of the parameters, we make use of Bayes factors, which are summary measures that provide an alternative to the $\mathrm{p}$-value for the ranking of associations, or the flagging of associations as "significant", (43). Large Bayes factors show strong evidence for the alternative hypothesis (similar to small $P$ values). Factors can be interpreted according to the values shown in online Supplementary Table S3. The $95 \%$ highest posterior density (HPD) interval is the shortest interval in parameter space that contains $95 \%$ of the posterior probability.

One advantage of the Bayesian approach is that it provides a natural resolution for the problem of data missing at random. In some cases, discipline data were missing from some databases for some students, as a result of inter-year group differences in school databases. As these data may naturally be assumed to be missing at random - that is, independent of any individual characteristics of the individual students - such likelihood-based inference is known to manage missing data well ${ }^{(44)}$. The time at risk for each pupil was adjusted to reflect missing data, as well as the fact that all pupils were not in the same databases and therefore could not be expected to have the same exposure. This approach also naturally incorporates the baseline measures in estimating the treatment effect. In doing so, it provides some correction for random differences in underlying rates between the placebo and treatment groups, a problem that turned out to be substantial in this case (see below).

School discipline records - stratification by number of baseline offences. Inspired by the recent work of Long and Benton $^{(45)}$, which seems to show a divergence of effect between subjects selected on the basis of their scores at baseline - they reported an improvement in the 'high impulsivity' at baseline group, and their plots seem to show an equally strong deterioration in the 'low impulsivity' group - we divided our subjects according to their number of disciplinary events in the baseline period. The 'high group' included the twenty-five subjects with more than three disciplinary events during the baseline period, and the 'low group' included the remaining 171 subjects.

We extended our Bayesian model to allow for different treatment effects in these two groups.

A detailed model description of the adjusted model can be found in the online Supplementary Material.

School discipline records - subgroup analysis with PUFA measurements. We considered the possibility that the effect of nutritional supplements might be proportional to individual changes in blood levels of the nutrients. Comparing the individual blood measurements with the behaviour changes could then provide more power to detect the effect. Approximately onefourth of the participants consented to provide blood samples from which erythrocyte PUFA and plasma levels of other
Table 1. Baseline participant characteristics (Numbers and percentages)

\begin{tabular}{|c|c|c|c|c|c|c|}
\hline \multirow[b]{2}{*}{ Baseline } & \multicolumn{2}{|c|}{ Active (n 98) } & \multicolumn{2}{|c|}{ Placebo (n 98) } & \multirow[b]{2}{*}{$\chi^{2}$} & \multirow[b]{2}{*}{$P$} \\
\hline & $n$ & $\%$ & $n$ & $\%$ & & \\
\hline \multicolumn{7}{|l|}{ Sex } \\
\hline Male & 43 & 44 & 55 & 56 & \multirow[t]{2}{*}{2.47} & \multirow[t]{2}{*}{0.12} \\
\hline Female & 55 & 56 & 43 & 44 & & \\
\hline \multicolumn{7}{|c|}{ Age (years) } \\
\hline 13 & 8 & 8 & 9 & 10 & \multirow[t]{4}{*}{$1 \cdot 16$} & \multirow[t]{4}{*}{0.76} \\
\hline 14 & 53 & 54 & 50 & 50 & & \\
\hline 15 & 37 & 38 & 38 & 38 & & \\
\hline 16 & 0 & 0 & 1 & 2 & & \\
\hline \multicolumn{7}{|l|}{ Year/grade } \\
\hline 9 & 12 & 12 & 14 & 14 & \multirow[t]{2}{*}{0.04} & \multirow[t]{2}{*}{0.83} \\
\hline 10 & 86 & 88 & 84 & 86 & & \\
\hline \multicolumn{7}{|l|}{ Cohort } \\
\hline 1 & 27 & 28 & 26 & 26 & \multirow[t]{4}{*}{1.25} & \multirow[t]{4}{*}{0.74} \\
\hline 2 & 37 & 38 & 35 & 36 & & \\
\hline 3 & 25 & 26 & 23 & 24 & & \\
\hline 4 & 9 & 8 & 14 & 14 & & \\
\hline
\end{tabular}

micronutrients were analysed. As before, detailed descriptions of the hazard rates used are given in the online Supplementary Material. The parameters $\alpha$ and $\alpha_{A}$ have the same interpretations as given in the section on the full analysis, but this model also included the parameter $\beta$ to measure the significance of the change in blood concentrations on behaviour. Again, negative values of $\beta$ would correspond to the predicted result that an increase in the blood concentration would reduce a pupil's offence rate. Positive values of $\beta$ would mean that an increase in blood concentration raises a pupil's offence rate.

\section{Results}

\section{Baseline measures}

At baseline, there were no significant differences in sex, age, year group or cohort between active and placebo groups (Table 1). Unfortunately, however, randomisation did not provide matched groups for baseline disciplinary infractions; the disciplinary rates were 2.5 times greater in the placebo group than in the active treatment group.

\section{Number of days in treatment and compliance}

The mean number of days in the study was $77 \cdot 1$ (SD 10.0) $d$ for the active group and $76 \cdot 1$ (sD 13.3) d for the placebo group that is, there was no significant difference between the groups $(P=0.56)$. The mean compliance rate was 88.4 (sD 10.9) \% for the active treatment and 88.5 (SD 8.8) \% for the placebo group; again this difference was not statistically significant $(P=0 \cdot 91)$.

\section{Dietary intake}

Food diaries were completed by most participants ( $n$ 117, active $n 66$, placebo $n 51$ ). These revealed no significant differences in baseline nutrient intake between the groups (online Supplementary Table S2). Mean number of days completed were different between groups as a result of a small number of 
individuals in the placebo group choosing to complete $<7 \mathrm{~d}$ in their diaries; however, this was not associated with differences in mean daily nutrient intake.

\section{Treatment guesses}

At baseline, the proportion of participants who guessed correctly in either group was not statistically different $\left(\chi^{2}=1.93\right.$; $P=0 \cdot 17$ ). However, after the treatment, the proportion of the children who guessed correctly differed between groups $\left(\chi^{2}=10 \cdot 24 ; P=0 \cdot 001\right)$, with 56 and $70 \%$ of students in the active and placebo group, respectively, correctly guessing their allocation. However, their teachers were unable to correctly guess the treatment allocations $\left(\chi^{2}=1 \cdot 31 ; P=0 \cdot 25\right)$.

\section{Erythrocyte fatty acid and micronutrient analysis}

Concentrations of erythrocyte membrane fatty acids expressed as percentages can be seen in Table 2 . The baseline EPA, DHA, total $n-3$ and the $n-3$ index were very low, whereas the total $n-6$ and $n$ 6: $n$-3 ratios were very high. All these PUFA status measures improved significantly after 12 weeks of active treatment - that is, EPA, DHA, total $n-3$ and the $n-3$ index increased, whereas total $n-6$ and the $n-6: n-3$ ratio decreased (all $P \leq 0 \cdot 0005$ ); this resulted in large treatment effects, as seen by differences between active and placebo groups post-intervention (all $P \leq 0.0005$ ). Similarly, plasma concentrations of folate, vitamin $\mathrm{C}$ and vitamin $\mathrm{D}$ increased significantly $(P \leq 0 \cdot 001)$ as a result of supplementation (Table 3). However, Fe and ferritin showed no significant changes. Other micronutrients of interest were unable to be measured in the blood as a result of technical limitations.

\section{Conners assessment}

With regard to the trial registration, this was stated as a primary outcome measure. The Conners' Teacher's mean scores are shown in Table 4. The individual scores were normally distributed. We used a one-way ANCOVA to test for an effect of the treatment on an individual's change in score from the pretreatment to the post-treatment test. The disruptive behaviour disorder subscale showed a significant post-intervention between-group difference $(P=0.02$; Table 4$)$. The treatment group improved (decreased $T$-score) slightly, whereas the placebo group's average $T$-score increased significantly $(P=0.005)$, meaning that their behaviour worsened. We did not anticipate changes in any other subscales, and none of our statistical tests show any significant changes within or between groups after the 12-week intervention.

An alternative approach is to perform $t$ tests directly on the changes in the Conners scores (post-pre). This showed a significant difference, between treatment groups, in the disruptive behaviour subscale $(P=0.009$; Fig. 2$)$, with no other significant differences.

\section{School discipline data - whole group analysis}

With regard to the trial registration, this was stated as a primary outcome measure. A small number of participants $(n 7)$ stopped

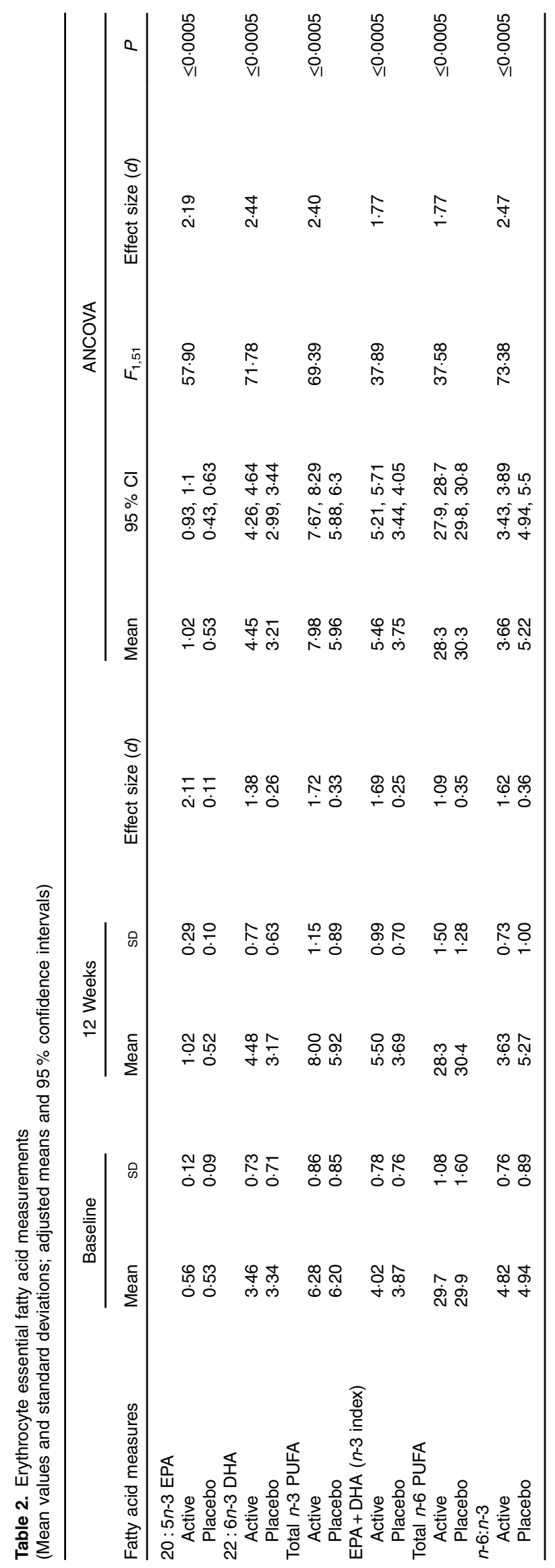




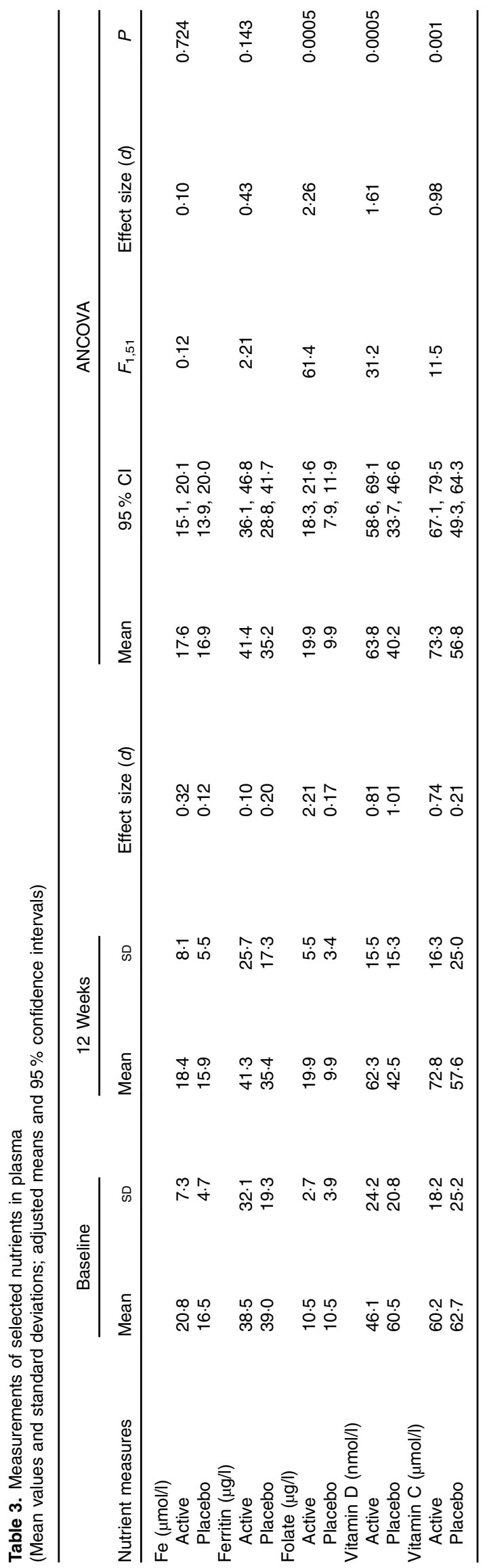

taking supplements before the end of the trial period (mean drop-out on treatment day 33 (SD 20)). These data were still used in an ITT analysis as disciplinary and compliance data were available for the period in which supplementation was taken.

The results obtained in an ITT analysis using all pupil data and fitting the model using the hazard rate is described in the online Supplementary Material (Table 5). Taking both groups together, the pupils demonstrated a multiplicative increase in the hazard rate of $e^{\alpha+\alpha A}$, which corresponds to a significant increase in disciplinary offending by $25 \%$. Actually, the treated group showed an additional increase in the hazard rate of $e^{\alpha A}$ ( $8.5 \%$ ), but this difference failed to reach statistical significance because of the high variability among pupils.

In other words, pupils in the placebo group increased their offence rates by 25 (95\% HPD 3; 48)\% during the treatment period, whereas pupils receiving the active treatment experienced a 34 (95\% HPD 2; 69) \% increase in their offence rates. Although the active group displayed a seemingly higher increase, there was more uncertainty in the estimate, and thus the difference was insignificant.

\section{School discipline data - stratification by the number of baseline offences}

After splitting the analysis into groups of high ( $>3$ disciplinary events during the baseline period) and low misbehaviour rates, we found that the median estimate of the parameter $\beta_{H A}$ was -0.674 , implying a decrease of 51 (95\% HPD 34; 62) \% in misbehaviour rate among those in the high-misbehaviour group who received the treatment, relative to those who received the placebo (Table 6). However, also the median estimate of the parameter $\beta_{L A}, 0.43$, implied an increase of 53 (95\% HPD 1; 137) \% in misbehaviour rate among those in the low-misbehaviour group who received the treatment, relative to those who received the placebo. We consider the result for the low-offending group to have more statistical reliability, as explained further in the 'Discussion' section.

We repeated the analysis using thresholds of 1, 2, 3, 4 and 5 baseline offences and observed similar results. Lower thresholds, by reducing the number of subjects in the low group, also reduced the statistical significance.

\section{School discipline data - subgroup analysis with participants who gave blood}

It seems plausible to suppose that effects of the supplements would be mediated by the amount by which blood levels change, although this may be masked by variability in individual responses to the supplements. If, for instance, a substantial change in blood levels of EPA or other micronutrients improved subjects' behaviour, this may be seen in only some of the treatment group. We looked for such an effect by fitting various models.

Our investigations confirmed that such a model could in principle improve the power to detect an effect that would be obscured in a simple comparison of placebo and treatment 
groups. Unfortunately, this potential improvement in power was overwhelmed by the reduction in sample size, because of the small number of subjects who agreed to give blood samples. Therefore, these models were prone to overfitting, and turned out to be highly sensitive to details of model choice and individual outliers in the data.

\section{Discussion}

The main conclusions from this work are as follows: (1) that teenagers from disadvantaged backgrounds tend to have very low blood levels of DHA, EPA and other micronutrients; (2) these can be effectively increased by taking appropriate supplements; (3) that increasing these levels may reduce these students' disruptive behaviour; and (4) this improvement may only translate to a reduction in disciplinary offences in those with a high initial level of infringements.

\section{Blood findings}

These children had extremely low levels of the $n$-3, DHA and EPA coupled with relatively high levels of $n-6$ (although the latter were still low by accepted standards). Although there are as yet no officially recommended levels of these fatty acids, the consensus is that ideally EPA and DHA should constitute $8 \%$ of total erythrocytes lipid composition ${ }^{(46)}$. In these adolescents, they amounted to only half of the ideal $-4 \%$. Although all nutrients are important ${ }^{(47)}, n-3$ are particularly so, especially when the brain is experiencing rapid growth during puberty and is at its most sensitive to insufficient supply. EPA has a more functional role in neural signalling in older subjects, whereas DHA has a more structural neurodevelopmental role in nerve membranes. Stevens et $a l .{ }^{(48)}$ found a correlation between improvements in conduct and $n-3$ PUFA levels. In addition, increased DHA was found to be associated with improvements in parent-rated behaviour ${ }^{(49)}$.

We hoped therefore that improvements in EPA and/or DHA levels in the blood would predict improvements in these adolescents on our behavioural measures. However, this was not seen, probably because of the small numbers of participants opting to provide a blood sample. Neither did increased levels of $\mathrm{Fe}$, ferritin, folate, vitamin $\mathrm{C}$ or vitamin $\mathrm{D}$ predict improvements, using the same models as for the $n-3$ PUFA. However, these potential associations deserve further investigation. The use of a less invasive technique such as finger prick may increase volunteer numbers.

Nevertheless, significant improvements in PUFA and micronutrient status were seen in the participants who agreed to provide blood. This finding confirms the improvements in PUFA status reported in many other studies - e.g. Hamazaki ${ }^{(30)}$, Voigt et $a l^{(27)}$ and Itomura et $a l^{(23)}$. Although we cannot be sure that this improvement led to improved fatty acid profiles in the brain, nevertheless Carver ${ }^{(50)}$ showed that there is a significant correlation between erythrocyte and neuronal $n$-3 PUFA content in adults, although this relationship failed to reach significance in subjects $<18$ years old. Importantly, the very low blood fatty acid (both $n-3$ and $n$-6) values at baseline of our participants suggest that these children did indeed have a very poor diet. Their low intakes of $n-3$ PUFA (Table 2) are consistent with the very low intake of 
Fig. 2. Conners subscales (changes in $T$-scores) post-treatment. ADHD, attention-deficit hyperactivity disorder.

Table 5. Treatment effect on school disciplinary incidents (Mean values, standard deviations and $95 \%$ highest posterior density interval (HPDI))

\begin{tabular}{lccccc}
\hline & Mean & SD & $95 \%$ HPDI & $H_{O}$ & Bayes factor \\
\hline$a$ & 0.22 & 0.092 & $0.040 ; 0.40$ & $a<0$ & 115 \\
$a_{A}$ & 0.07 & 0.156 & $-0.23 ; 0.38$ & $a_{A}<0$ & 2.04 \\
\hline
\end{tabular}

Table 6. Parameter estimates resulting from an MCMC chain of 100000 iterations*

(Mean values, standard deviations and $95 \%$ highest posterior density intervals (HPDI))

\begin{tabular}{lrcccc}
\hline & Mean & SD & $95 \%$ HPDI & $H_{0}$ & Bayes factor \\
\hline$K_{H}$ & 3.326 & 0.136 & $3.058 ; 3.588$ & & \\
$a_{H}$ & 0.354 & 0.092 & $0.170 ; 0.531$ & $a_{H}<0$ & $>1000$ \\
$a_{L}$ & 0.209 & 0.209 & $-0.208 ; 0.609$ & $a_{L}<0$ & 5.27 \\
$\beta_{H A}$ & -0.676 & 0.142 & $-0.957 ; 0.402$ & $\beta_{H A}>0$ & $>1000$ \\
$\beta_{L A}$ & 0.428 & 0.216 & $0.012 ; 0.858$ & $\beta_{L A}<0$ & 42.44 \\
$\lambda$ & 0.010 & 0.001 & $0.007 ; 0.012$ & & \\
\hline
\end{tabular}

* A large Bayes factor indicates evidence for the alternative hypothesis. The HPDI is similar to a $\mathrm{Cl}$, but more straightforward to interpret. According to the model, the probability is $95 \%$ that the true parameter lies within this interval. Thus, the parameter $\beta_{L A}$, measuring the change in offence rate among the baseline low-offending group, is $95 \%$ likely to be between 0.012 and 0.858 . In particular, a two-tailed test at the 0.05 level would reject the hypothesis that this effect is 0 .

foods containing long-chain $n-3$ PUFA in the typical British diet ${ }^{(37)}$. Their very low $n-3$ index would predict diminished protection from $\mathrm{CHD}$ in adulthood ${ }^{(51)}$. Although we achieved significant improvements post-treatment, the $n-3$ index did not reach the $8 \%$, which is associated with the greatest protection against $\mathrm{CHD}$. This may suggest that the duration of our supplementation was insufficient, and this could help explain our disappointing results with respect to the children's disciplinary offences. Future studies might benefit from addressing these points. Nonetheless, the results of the fatty acid analysis provide normative values of erythrocyte $n$-3 PUFA in typical underprivileged UK adolescents.

Limitations of the micronutrient analysis. We studied typically developing adolescent schoolchildren from a particularly underprivileged demographic and environment. As such it was expected and demonstrated that they would have a relatively low intake of many micronutrients. Our intention was to investigate all of these. However, technical issues in blood storage and processing did not permit some of them. Nonetheless, the micronutrients that we were able to analyse showed that supplementation improved blood concentrations in almost all cases. However, as with the PUFA, it is not possible to ascertain whether this improvement led to improvements in brain tissue levels.

\section{Disruptive behaviour}

Our results suggest that there may be a favourable effect of $n-3$ PUFA, vitamin and mineral supplementation on disruptive behaviour in these typically developing adolescent schoolchildren, as assessed by the Conners Teacher Rating Scale, but it was probably small. The disruptive behaviour subscale scores improved slightly in the treated group, whereas that of the placebo group worsened considerably. The Conners questionnaire is a standardised, valid and reliable assessment, and it appears to be sensitive enough to detect modest treatment effects, even when baseline scores were within a non-clinical range. The teachers were unable to guess which pupils had been allocated active or placebo, and thus their assessments were unlikely to have been affected by their guesses; this strengthens our confidence in the reliability of the Conners data. 
As expected, no effect was seen in the other Conners subscale measurements. Kirby et $a l^{(17)}$ used a Strengths and Difficulties Questionnaire to measure, inter alia, conduct problems and prosocial behaviour. They found that treatment left the children's parent-rated prosocial behaviour scores unchanged, whereas in the placebo group these worsened, yielding a significant advantage to the treated group. Similar between-group patterns were seen by Itomura et al. ${ }^{(23)}$ and Hamazaki et al. ${ }^{(21)}$. Future work could consider measuring other social outcome measures such as callous and unemotional traits ${ }^{(52)}$ or the Reactive and Proactive Questionnaire ${ }^{(53)}$

Considering the $P$ value for the Connors disruptive behaviour subscale in isolation might reasonably be challenged, as it selects a single subscale for testing, ignoring four others that showed no significant change. Considering all subscales together as multiple tests of a single null hypothesis, a single $P$ value of 0.02 (from the ANCOVA) would not necessarily be statistically significant, as a Bonferroni correction would raise the $P$ value to the range of a trend $(P=0 \cdot 1)$, whereas two-sample $t$ tests would still yield a marginally significant treatment effect on the Conners scale (Bonferroni corrected from $P=0.009$ to $P=0.045$ ). At the very least, this suggests that the result be treated with some caution. On the other hand, the disruptive behaviour subscale was the one that was relevant for this study, and that we sought to measure. The fact that four additional subscales come along as unavoidable adjuncts of this tool, and could, in principle, themselves be investigated for significant treatment effects, does not detract from the significant treatment effect observed in the target measure.

\section{Disciplinary data}

When the school discipline records of all the volunteers were analysed together, we could see no positive behavioural effect of the supplementation. Over each school term the behaviour of both groups worsened significantly; unfortunately this termly pattern is common in schools. Thus, all the pupils, taken together, increased their offence rates by 25 (95\% HPD 3; 48) \%, and it appears that receiving the active treatment had little effect on offending as measured by these disciplinary records. Thus, our disciplinary findings did not confirm the findings of Schoenthaler and Bier ${ }^{(13)}$ in schoolchildren, or those of Gesch et al. ${ }^{(15)}$, and Zaalberg et $a l^{(16)}$, in prisoners; all these studies found reduced offending behaviour after supplementation. However their baseline levels of offending were higher, and different treatments were used in each of them. Schoenthaler gave vitamins and minerals alone, Gesch administered vitamins, minerals and PUFA (predominantly $n$-6) and Zaalberg used vitamins, minerals and PUFA (predominantly $n$-3). As a comparison, other than a lower EFA content, our study used a similar formulation to Zaalberg in that it contained doses of vitamins and minerals close to reference levels.

A recent study ${ }^{(45)}$ showed an improvement in laboratory-based measures of aggression and impulsivity in young adults upon DHA supplementation, particularly in those with higher baseline levels of impulsivity. Indeed, some measures in that study seemed to show deterioration among those receiving DHA supplementation if they started with lower baseline levels of impulsivity. Our results from analysis of the school discipline data (Table 6) show a similar pattern, and they are suggestive of a large treatment effect $v$. placebo only in children with high misbehaviour rates at baseline. This pattern is what one might expect if the supplements reduced the impulsivity and lack of executive control seen particularly in the high offenders.

However, this conclusion should be viewed with caution. The high-misbehaviour group consisted of only a small number of individuals (twenty-five) with a very large number of offences (as many as thirty-three at baseline). The estimated effect size may thus be dominated by effects of regression to the mean, which is highly dependent upon the choice of statistical model. We have no strong theoretical basis for choosing any particular model for this setting, and the small number of subjects makes empirical estimation impossible. This uncertainty is exacerbated by the difference in distribution between the treatment and control groups, which was unfortunately concentrated entirely in this high group. Despite random allocation of active or placebo treatment, the overall disciplinary rates in the two groups were very different during the baseline period; the pupils in the active treatment group had an average offence rate of only 0.017 , whereas those in the placebo group had an average rate of 0.042 - that is, it was 2.5 times greater than that of the active group. Using ten million bootstrap samples, we found that the probability of achieving this allocation at random was only 0.009 - that is, approximately 1 in 100; yet it happened in our case. Thus, most of the prolific offenders ended up in the placebo group, giving us less information to estimate parameters associated with the active treatment - hence the greater uncertainty around $\alpha_{A}$.

One virtue of the statistical procedures that we have used here, which control for individual baseline observations, is that our main parameter estimates should remain unbiased even in the face of such an unbalanced allocation. The CI, however, became broader than they would otherwise have been, increasing our uncertainty. The unbalanced randomisation also exacerbates model uncertainties. A simple randomisation procedure was used in this study, and thus future studies might benefit from using stratified randomisation to ensure equal distribution of prolific offenders in each treatment group.

In addition, we had methodological problems with the disciplinary records, which were not standardised or validated measures of behaviour and consisted of four different registers with different criteria. Despite their having being used in similar trials in schools and prisons, and that they provide an ecologically valid behavioural log, it may be that their piecemeal application here as a measure of behavioural change was inappropriate, leading to the contradictory results. Inspired by results reported elsewhere ${ }^{(45)}$, rather than only after looking at our data, we decided to stratify the participants by their baseline levels of misbehaviour, and found different outcomes for those with high $v$. low levels. Thus, our failure to see any advantage in the whole treated group may have been the result of combining one group with low and another with high misbehaviour rates at baseline, so that the treatment effects opposed each other. It may also reflect difficult-to-control statistical fluctuations in the small number of individuals who were responsible for the largest number of offences, exacerbated by the unfortunately unbalanced randomisation. 
Any analysis that includes the high-misbehaviour group is subject to unpredictable effects of regression to the mean, acting differentially between the treatment and control groups. Indeed, one might argue that the main advantage of stratifying by baseline behaviour was to remove this small but very problematic subset from the model.

Our estimates of the treatment effect in the remaining low-offence group (nearly $90 \%$ of the original study population) is more reliable statistically, as the treatment and placebo groups within it had very similar distributions of offences, and the estimates are not being driven by a small number of individuals with large numbers of offences. Proper estimation of the treatment effect in the high-misbehaviour subpopulation would require a larger sample size, and greater attention to matching the treatment and placebo groups at baseline. The direction of the effect in children in the low misbehaviour group was opposite to that expected, although consistent with the recent results of Long and Benton ${ }^{(45)}$. We speculate that supplementation in this group might have increased their general alertness and energy, which may have made them uncharacteristically disrespectful, and that this may have been treated as misbehaviour by the teachers. Therefore, supplementation could be an effective therapeutic approach only in individuals who have high rates of misbehaviour, but not so in those whose behaviour is more typical.

Thus, our results suggest that active supplementation might have had a moderating effect against an increase in aberrant behaviour, when assessed using the Conners standardised tests, but this is far less clear using the school disciplinary records.

\section{Blinding}

Interestingly, some participants were able to guess to which treatment they had been allocated after the supplementation period. Thus, a partial breaking of the blind might have occurred. As the supplements were well matched for colour, odour and flavour, we suggest that these children had felt the benefit of their enhanced micronutrient levels (or otherwise, if allocated placebo) and thus correctly identified their group. In the study by Zaalberg et al. ${ }^{(16)}$, the participants were also able to guess correctly for similar reasons. We cannot know whether this resulted in pupils purposely altering their behaviour, but it would seem unlikely.

\section{Conclusion}

Previous studies in prisoners or children with dyspraxia or ADHD have limited applicability to typically developing children. We attempted to address this issue by recruiting children with no formal diagnosis of any neurodevelopmental condition. We found suggestive evidence that broad-spectrum vitamin, mineral and $n-3$ supplementation might have a protective effect against anti-social behaviour, when assessed using Conners scales. We also found some evidence that supplementation may decrease misbehaviour among children whose prior rates were high, yet may increase it in children whose baseline rates were low. Thus, if aberrant behaviour is a manifestation of non-optimal nutrient intake, these findings warrant further investigation with larger numbers, increased nutrient dose and stratifying offending rates.

\section{Acknowledgements}

The authors thank the children of Robert Clack School, Dagenham, UK for volunteering, and Sir Paul Grant, Phil Akerman and staff for their generous support. The authors also thank Louise Gillam for food diary analysis, Anna Pitt and Anli Wang for their assistance, Gordon Bell and James Dick (University of Stirling) for chemical analysis of PUFA and Lorna Cox and Chris Bates (MRC HNR Cambridge) for chemical analysis of micronutrients. Nutrient supplements were generously provided by Vitabiotics Ltd (UK). However, they played no part in the design, execution, analysis or writing.

This work was supported by a grant from the Esmée Fairbairn Foundation (grant ref: 09-2343) and commissioned by the Institute for Food Brain and Behaviour (IFBB). Supplementary support was received from the E. P. Abraham, IFBB and DRT Trusts. The funders had no role in the design, execution, analysis or writing of this article.

J. D. T., T. S.-A. and J. F. S. designed the research. J. D. T. and T. S.-A. conducted the research. J. F. S. was responsible for oversight. D. S. and D. W. B. carried out the Bayesian statistical analysis for the discipline report data. J. D. T. carried out statistical analysis for other measures. J. D. T. wrote the manuscript with others contributing to subsequent drafts. All authors approved the manuscript.

There are no conflicts of interest to declare.

\section{Supplementary material}

For supplementary material/s referred to in this article, please visit http://dx.doi.org/doi:10.1017/S0007114515004390

\section{References}

1. Galler JR (2005) Behavioral effects of childhood malnutrition. Am J Psychiatry 162, 1760-1761.

2. Benton D (2007) The impact of diet on anti-social, violent and criminal behaviour. Neurosci Biobehav Rev 31, 752-774.

3. Iribarren C, Markovitz JH, Jacobs DR, et al. (2004) Dietary intake of $n-3, n-6$ fatty acids and fish: relationship with hostility in young adults [mdash] the CARDIA study. Eur J Clin Nutr 58, 24-31.

4. Hibbeln JR, Ferguson TA \& Blasbalg TL (2006) Omega-3 fatty acid deficiencies in neurodevelopment, aggression and autonomic dysregulation: opportunities for intervention. Int Rev Psychiatry 18, 107-118.

5. Youdim KA, Martin A \& Joseph JA (2000) Essential fatty acids and the brain: possible health implications. Int J Dev Neurosci 18, 383-399.

6. Simopoulos AP (2011) Evolutionary aspects of diet: the omega-6/omega-3 ratio and the brain. Mol Neurobiol 44, 203-215.

7. Phillis JW, Horrocks LA \& Farooqui AA (2006) Cyclooxygenases, lipoxygenases, and epoxygenases in CNS: their role and involvement in neurological disorders. Brain Res Rev $\mathbf{5 2}$, 201-243. 
8. Rucklidge JJ \& Kaplan BJ (2013) Broad-spectrum micronutrient formulas for the treatment of psychiatric symptoms: a systematic review. Expert Rev Neurother 13, 49-73.

9. Ames BN, Eslson-Schwab I \& Silver EA (2002) High-dose vitamin therapy stimulates variant enzymes with decreased coenzyme binding affinity (increased $\mathrm{K}(\mathrm{m})$ ): relevance to genetic disease and polymorphisms. Am J Clin Nutr 75, 616-658.

10. Frensham LJ, Bryan J \& Parletta N (2012) Influences of micronutrient and omega- 3 fatty acid supplementation on cognition, learning, and behaviour: methodological considerations and implications for children and adolescents in developed societies. Nutr Rev 70, 594-610.

11. Grima NA, Pase MP, Macpherson H, et al. (2012) The effects of multivitamins on cognitive performance: a systematic review and meta-analysis. J Alzheimers Dis 29, 561-569.

12. Parletta N, Milte CM \& Meyer BJ (2013) Nutritional modulation of cognitive function and mental health. J Nutr Biochem $\mathbf{2 4}$, $725-743$.

13. Schoenthaler SJ \& Bier ID (2000) The effect of vitamin-mineral supplementation on juvenile delinquincy among American Schoolchildren: a randomised, double-blind placebocontrolled trial. J Altern Complement Med 6, 7-17.

14. Schoenthaler S, Amos S, Doraz W, et al. (1997) The effect of randomized vitamin-mineral supplementation on violent and non-violent antisocial behavior among incarcerated juveniles. J Nutr Environ Med 7, 343-352.

15. Gesch CB, Hammond SM, Hampson SE, et al. (2002) Influence of supplementary vitamins, minerals and essential fatty acids on the antisocial behaviour of young adult prisoners: randomised, placebo-controlled trial. Br J Psychiatry 181, 22-28.

16. Zaalberg A, Nijman H, Bulten E, et al. (2010) Effects of nutritional supplements on aggression, rule-breaking, and psychopathology among young adult prisoners. Aggress Behav 36, 117-126.

17. Kirby A, Woodward A, Jackson S, et al. (2010) A double-blind, placebo-controlled study investigating the effects of omega-3 supplementation in children aged 8-10 years from a mainstream school population. Res Dev Disabil 31, 718-730.

18. Raine A, Portnoy J, Liu J, et al. (2015) Reduction in behavior problems with omega-3 supplementation in children aged 8-16 years: a randomized, double-blind, placebo-controlled, stratified, parallel-group trial. J Child Psychol Psychiatry 56, 509-520.

19. Stevens L, Zentall S, Deck J, et al. (1995) Essential fatty acid metabolism in boys with attention-deficit hyperactivity disorder. Am J Clin Nutr 62, 761-768.

20. Stevens LJ, Zentall SS, Abate ML, et al. (1996) Omega-3 fatty acids in boys with behavior, learning, and health problems. Physiol Behav 59, 915-920.

21. Hamazaki T, Sawazaki S, Itomura M, et al. (1996) The effect of docosahexaenoic acid on aggression in young adults. A placebo-controlled double-blind study. J Clin Invest 97, 1129.

22. Bilici M, YildIrIm F, Kandil S, et al. (2004) Double-blind, placebocontrolled study of zinc sulfate in the treatment of attention deficit hyperactivity disorder. Prog Neuropsychopharmacol Biol Psychiatry 28, 181-190.

23. Itomura M, Hamazaki K, Sawazaki S, et al. (2005) The effect of fish oil on physical aggression in schoolchildren - a randomized, double-blind, placebo-controlled trial. J Nutr Biochem 16, 163-171.

24. Richardson AJ \& Puri BK (2002) A randomized double-blind, placebo-controlled study of the effects of supplementation with highly unsaturated fatty acids on ADHD-related symptoms in children with specific learning difficulties. Prog Neuropsychopharmacol Biol Psychiatry 26, 233-239.
25. Sinn N \& Bryan J (2007) Effect of supplementation with polyunsaturated fatty acids and micronutrients on learning and behavior problems associated with child ADHD. J Dev Behav Pediatr 28, 82-91.

26. Gustafsson PA, Birberg-Thornberg U, Duchén K, et al. (2010) EPA supplementation improves teacher-rated behaviour and oppositional symptoms in children with ADHD. Acta Paediatr 99, $1540-1549$.

27. Voigt RG, Llorente AM, Jensen CL, et al. (2001) A randomized, double-blind, placebo-controlled trial of docosahexaenoic acid supplementation in children with attention-deficit/ hyperactivity disorder. J Pediatr 139, 189-196.

28. Hirayama S, Hamazaki T \& Terasawa K (2004) Effect of docosahexaenoic acid-containing food administration on symptoms of attention-deficit//hyperactivity disorder [mdash] a placebocontrolled double-blind study. Eur J Clin Nutr 58, 467-473.

29. Rucklidge JJ, Frampton CM, Gorman B, et al. (2014) Vitaminmineral treatment of attention-deficit hyperactivity disorder in adults: double blind randomised placebo-controlled trial. Br J Psychiatry 204, 306-315.

30. Hamazaki K (2008) The effects of docosahexaenoic acid-rich fish oil on behavior, school attendance rate and malaria infection in school children - a double-blind, randomized, placebo-controlled trial in Lampung, Indonesia. Asia Pacific J Clin Nutr 17, 258-263.

31. Hamazaki T, Sawazaki S, Nagao Y, et al. (1998) Docosahexaenoic acid does not affect aggression of normal volunteers under nonstressful conditions. A randomized, placebo-controlled, double-blind study. Lipids 33, 663-667.

32. Konofal E, Lecendreux M, Deron J, et al. (2008) Effects of iron supplementation on attention deficit hyperactivity disorder in children. Pediatr Neurol 38, 20-26.

33. Mousain-Bosc M, Roche M, Polge A, et al. (2006) Improvement of neurobehavioral disorders in children supplemented with magnesium-vitamin B6. I. Attention deficit hyperactivity disorders. Magnes Res 19, 46-52.

34. Starobrat-Hermelin B \& Kozielec T (1997) The effects of magnesium physiological supplementation on hyperactivity in children with attention deficit hyperactivity disorder (ADHD). Positive response to magnesium oral loading test. Magnes Res 10, 149-156.

35. Benton D, Haller J \& Fordy J (1995) Vitamin supplementation for 1 year improves mood. Neuropsychobiology 32, 98-105.

36. Nelson M, Erens B, Bates B, et al. (2007) Low Income Diet and Nutrition Survey. Norwich: The Stationery Office.

37. Whitton C, Nicholson SK, Roberts C, et al. (2011) National Diet and Nutrition Survey: UK food consumption and nutrient intakes from the first year of the rolling programme and comparisons with previous surveys. Br J Nutr 106, 1899-1914.

38. Benton D (2013) To establish the parameters of optimal nutrition do we need to consider psychological in addition to physiological parameters? Mol Nutr Food Res 57, 6-19.

39. Committee on Medical Aspects of Food (1991) Dietary Reference Values for Food Energy and Nutrients for the United Kingdom. Report on Health and Social Subjects, vol. 41. Norwich: The Stationery Office.

40. Bell JG, Mackinlay EE, Dick JR, et al. (2011) Using a fingertip whole blood sample for rapid fatty acid measurement: method validation and correlation with erythrocyte polar lipid compositions in UK subjects. Br J Nutr 106, 1408-1415.

41. Conners CK (2008) Conners Comprehensive Behaviour Rating Scales Manual. North Tonawanda, NY: Multi-Health Systems Inc.

42. Richardson AJ, Burton JR, Sewell RP, et al. (2012) Docosahexaenoic acid for reading, cognition and behavior in children aged 7-9 years: a randomized, controlled trial (the DOLAB Study). PLOS ONE 7, e43909. 
43. Wakefield J (2008) Bayes factors for genome-wide association studies: comparison with P-values. Genet Epidemiol 33, 79-86.

44. Little R \& Rubin D (2002) Statistical Analysis with Missing Data, 2nd ed., vol. 4. New York, NY: Wiley.

45. Long SJ \& Benton D (2013) A double-blind trial of the effect of docosahexaenoic acid and vitamin and mineral supplementation on aggression, impulsivity, and stress. Hum Psychopharmacol 28, 238-247.

46. Meyer BJ, Byrne MK, Collier C, et al. (2015) Baseline omega-3 index correlates with aggressive and attention deficit disorder behaviours in adult prisoners. PLOS ONE 10, e0120220.

47. Mazza M, Pomponi M, Janiri L, et al. (2007) Omega-3 fatty acids and antioxidants in neurological and psychiatric diseases: an overview. Prog Neuropsychopharmacol Biol Psychiatry 31, $12-26$.

48. Stevens L, Zhang W, Peck L, et al. (2003) EFA supplementation in children with inattention, hyperactivity, and other disruptive behaviors. Lipids 38, 1007-1021.
49. Milte CM, Parletta N, Buckley JD, et al. (2012) Eicosapentaenoic and docosahexaenoic acids, cognition, and behavior in children with attention-deficit/hyperactivity disorder: a randomized controlled trial. Nutrition 28, 670-677.

50. Carver JD, Benford VJ, Han B, et al. (2001) The relationship between age and the fatty acid composition of cerebral cortex and erythrocytes in human subjects. Brain Res Bull 56, 79-85.

51. Harris WS \& von Schacky C (2004) The Omega-3 Index: a new risk factor for death from coronary heart disease? Prev Med 39 , 212-220.

52. Frick PJ, Ray JV, TL C, et al. (2014) Annual research review: a developmental psychopathology approach to understanding callous-unemotional traits in children and adolescents with serious conduct problems. J Child Psychol Psychiatry 55, 532-548.

53. Raine A, Dodge K, Loeber R, et al. (2006) The reactive-proactive aggression questionnaire: differential correlates of reactive and proactive aggression in adolescent boys. Aggress Behav 32, 159-171. 